\title{
Normal S cone electroretinogram b-wave in Oguchi's disease
}

Shuichi Yamamoto, Masanori Hayashi, Shinobu Takeuchi, Yutaka Shirao, Katsutoshi Kita, Kazuo Kawasaki

\begin{abstract}
Aim-The short wavelength sensitive (S) cone electroretinograms (ERGs) were examined in two patients with Oguchi's disease to study $\mathbf{S}$ cone function.

Methods-Ganzfeld colour flashes under bright white background illumination were used to elicit $S$ cone, and mixed long (L) and middle (M) wavelength sensitive cone ERGs.

Results-The S cone ERG b-wave was normal with short wavelength stimuli with normal $L$ and $M$ cone responses in both patients.

Conclusions-These ERG results indicate that the $S$ cone system as well as the $L$ and $M$ cone system is not defective in Oguchi's disease, while the $\mathbf{S}$ cone responses are not detectable in the complete type of congenital stationary night blindness as reported previously. The results imply that there is a significant difference in the way the $S$ cone system is affected in different forms of stationary night blindness, and that the $S$ cone and rod do not share the same arrestin system.

(Br f Ophthalmol 1997;81:1043-1045)
\end{abstract}

Oguchi's disease is a stationary form of congenital night blindness, in which a characteristic golden yellow or grey-white discoloration of the fundus and elevated dark adaptation threshold can be normalised after prolonged dark adaptation. ${ }^{1-4}$ Many investigators have postulated that this disorder is due to retarded resynthesis of visual pigments; however, normal rhodopsin kinetics have been reported and defects in bipolar cell layer have been suggested. ${ }^{5}$ Recently a homozygous deletion in the arrestin gene has been detected in Japanese patients and abnormalities in the rod phototransduction have been implicated. ${ }^{6}$ In the complete type of Schubert-Bornschein type congenital stationary night blindness (CSNB), in which exclusive defects of the 'on' pathway in the bipolar region have been postulated, the short wavelength sensitive (S) cone electroretinogram (ERG) b-wave is not detectable. ${ }^{8}{ }^{9}$ We now report two patients with Oguchi's disease whose S cone ERGs were normal.

Patients and methods

REPORT OF CASES

Case 1

A 35-year-old woman complained of night blindness of long duration. There was no con- sanguinity. No other family member has noticed night blindness. Her visual acuity was $20 / 25$ with a correction of -4.0 dioptres in both eyes. Ophthalmoscopically, a golden yellow glistening colour was observed throughout the retina in both eyes. Retinal vessels stood out in marked contrast. This fundus appearance became almost normal after 2 hours of dark adaptation confirming the presence of a Mizuo's phenomenon. She had normal colour vision on the Farnsworth Panel D-15. A molecular genetic study has not yet been performed.

Case 2

A 55-year-old woman also complained of night blindness since early childhood. Her parents were not consanguineous. The family history was not remarkable. Her visual acuity was 20/20 without correction in both eyes. On ophthalmoscopic examination, a golden yellow, metallic reflex was observed throughout both fundi. The Mizuo's phenomenon was also observed at the end of 12 hours of dark adaptation. Dark adaptometry showed that a normal rod final threshold was reached only after 12 hours of dark adaptation. Her colour vision tested by the Panel D-15 was also normal. Molecular genetic analysis has not been performed.

\section{ELECTRORETINOGRAPHY}

The method for ERG recordings was previously described. ${ }^{8}$ The ERGs were recorded using a Burian-Allen contact lens electrode. The patients' eyes were fully dilated with tropicamide eyedrops. A Ganzfeld stimulator provided full field flash stimuli and white background illumination. Light flashes were produced by a Grass PS22C strobe that has been placed into a metal box mounted on the Ganzfeld above the head of the subject. The white flash intensity was $5.0 \mathrm{cds} / \mathrm{m}^{2}$. Cone ERGs were recorded to white stimuli presented at $5 \mathrm{~Hz}$ on a white background light (50 $\left.\mathrm{cd} / \mathrm{m}^{2}\right)$. Kodak Wratten colour filters were placed before the strobe in order to obtain defined colour stimuli. The following filters were used on the same white background illumination: $98(450 \mathrm{~nm}), 48(471 \mathrm{~nm}), 61$ $(534 \mathrm{~nm}), 21(593 \mathrm{~nm})$, and $29(633 \mathrm{~nm})$. We routinely obtained responses using the maximum flash intensity available at $450 \mathrm{~nm}$ and then dimmed the other stimuli with neutral density filters to produce approximately equal $\mathrm{L}, \mathrm{M}$ cone b-wave, because all longer wavelength stimuli had more effective energy for the $\mathrm{L}$ and $\mathrm{M}$ cones. The stimulus rate was $5 \mathrm{~Hz}$ 


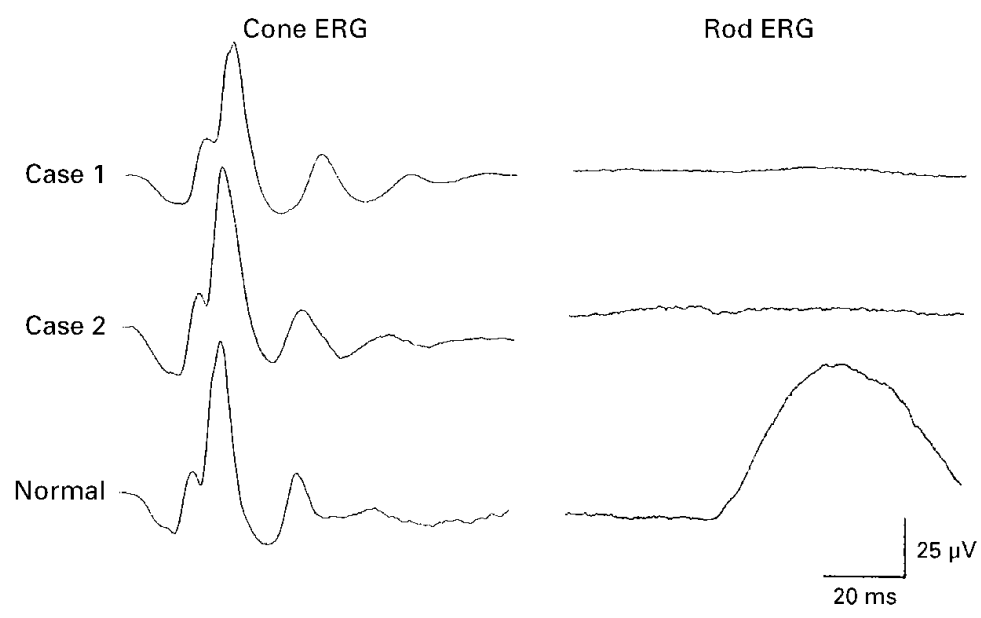

Figure 1 Cone ERGs to white flashes on a bright white background illumination (left), and averaged rod ERGs to dim blue flashes (right), after 2 hours of dark adaptation in case 1, after 12 hours of dark adaptation in case 2, and after 30 minutes of dark adaptation in a normal subject.

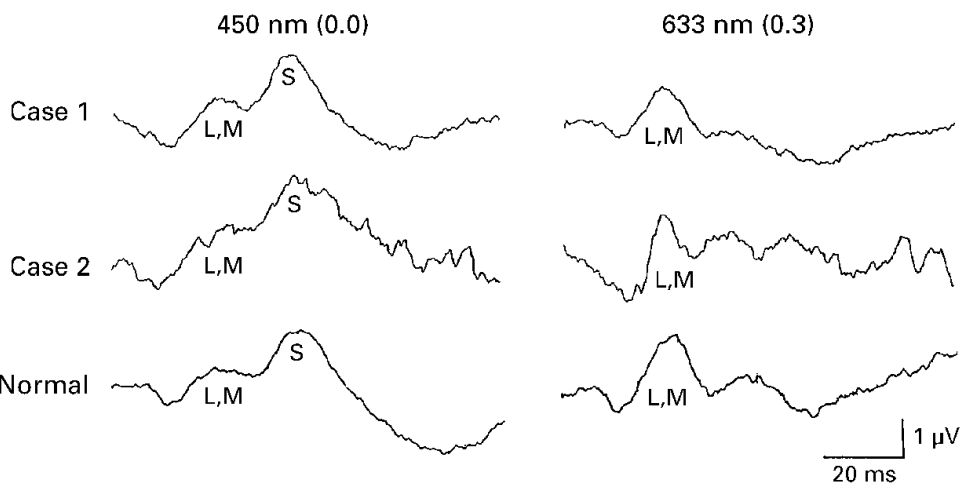

Figure 2 Cone ERGs to $450 \mathrm{~nm}$ stimuli (left) and to $633 \mathrm{~nm}$ stimuli (right) were recorded in the presence of a bright white adapting light. Numbers in parentheses indicate the neutral density filter used to adjust the energies of the flashes to produce approximately equal early ( $L, M$ cone) $b$-waves. $S$ signifies the $S$ cone $b$-wave, and $L, M$ indicates the $L$, $M$ cone b-wave. rod threshold only after prolonged dark adaptation, and undetectable averaged rod ERGs. Although Gouras ${ }^{4}$ reported that the first response to a single flash after dark adaptation showed the presence of rods, we could not detect the rod response because of an artefact caused by a blink in both patients. The cone ERGs to white stimuli were normal in our patients, as have been reported previously. ${ }^{34}$ The $S$ cone ERG elicited by short wavelength flashes was also normal, implying that the $S$ cone pathway was not affected in Oguchi's disease.

Early investigators had thought that an abnormality in the resynthesis of rhodopsin might be the reason for the prolonged dark adaptation in Oguchi's disease. However, Carr and Ripps ${ }^{5}$ reported that the concentration and regenerative properties of rhodopsin, tested by means of fundus reflectometry in one patient with Oguchi's disease, were entirely normal, suggesting that this disease might be related to the abnormalities in the bipolar cell region. Such a defect would imply that normal rod a-waves might be detectable in this disease but this has not been described. Recently, a homozygous defect in the arrestin gene has been discovered in Japanese patients with Oguchi's disease. ${ }^{67}$ Arrestin is known to bind to phosphorylated rhodopsin and to prevent it from activating transducin. These authors suggested that arrestin deficiency would result in prolonged activation of transducin and rod cGMP specific phosphodiesterase upon light stimulation of photoreceptors, which delay normal dark adaptation.?

It is well known that the rod system has only 'on' bipolars and involves an intermediary amacrine cell pathway. In the S cone pathway, there has been some evidence that there is a preponderance of S cone 'on' units at the ganglion cell level. ${ }^{10}$ We have reported that the $S$ cone ERG b-wave is undetectable, as well as the rod b-wave, in the complete type of CSNB. ${ }^{8}$ The defect in the complete type of CSNB may involve the 'on' pathway exclusively and therefore have a more profound effect on both the $S$ cone and rod bipolar systems. ${ }^{11}$ A similar type of acquired nyctalopia has been described in the so called cutaneous melanoma syndrome, ${ }^{12}$ which also lacks an $\mathrm{S}$ cone ERG. ${ }^{9}$ In this case immunohistochemistry reveals the bipolar cells as the site of the defect. ${ }^{13}$ However, the $S$ cone ERG b-waves are normal in patients with Oguchi's disease, implying that the $S$ cone bipolar system is not affected in this disease. This clearly distinguishes it from the complete type of CSNB. The $S$ cone ERG may be a useful tool to assess cone 'on' bipolar function and to distinguish different forms of congenital night blindness. Our results also imply that rods and $\mathrm{S}$ cones do not share the same arrestin system, although similar physiological properties have been reported in these photoreceptors in terms of slow response time and 'on' pathway predominance.

The authors are grateful to Peter Gouras, MD, for helpful comments on the manuscript.

Our patients demonstrated a characteristic golden yellow, glistening fundus colour associated with the Mizuo's phenomenon, normal 
1 Oguchi C. Über eine Fall von eigenartigen Hemeralopie. Acta Soc Ophthalmol fpn 1907;11:123-34.

2 Mizuo G, Nakamura B. Das Wesen von Oguchischen Krankheit und eine neue Kenntnis über Dunkeladaptation. Acta Soc Ophthalmol fpn 1914;18:73-126.

3 Carr RE, Gouras P. Oguchi's disease. Arch Ophthalmol 1965;73:646-56.

4 Gouras P. Electrophysiology: some basic principles. Invest Ophthalmol Vis Sci 1970;9:557-69.

5 Carr RE, Ripps H. Rhodopsin kinetics and rod adaptation in Oguchi's disease. Invest Ophthalmol Vis Sci 1967;6:42636.

6 Maw MA, John S, Jablonka S, Müller B, Kumaramanickavel G, Oehlmann R, et al. Oguchi's disease: suggestion of linkon chromosome $2 \mathrm{q}$. $f$ Med Genet 1995;32:396-8.

7 Fuchs S, Nakazawa M, Maw M, Tamai M, Oguchi Y, Gal A. A homozygous 1-base pair deletion in the arrestin gene is a frequent cause of Oguchi disease in Japanese. Nature Genet 1995;10:360-2.
8 Kamiyama M, Yamamoto S, Nitta K, Hayasaka S. Undetectable S-cone electroretinogram b-wave in complete station-

MacKay CJ, Seiki M, Gouras P, Roy M. Congenital and MacKay CJ, Seiki M, Gouras P, Roy M. Congenital and
acquired nyctalopias eliminate the S-cone ERG without disturbing color vision. Invest Ophthalmol Vis Sci 1995;36: S925.

$10 \mathrm{Kolb} \mathrm{H}$, Lipetz LE. The anatomical basis for color vision in the vertebrate retina. In: Gouras P, ed. The perception of colour. London: MacMillan Press, 1991:128-45.

11 Miyake Y, Yagasaki K, Horiguchi M, Kawase Y. On- and off-responses in photopic electroretinogram in complete and incomplete types of congenital stationary night blindness. Fpn f Ophthalmol 1987;31:81-7.

12 Berson EL, Lessell S. Paraneoplastic night blindness with malignant melanoma. Am f Ophthalmol 1988;106:307-11.

13 Weinstein JM, Kelman SE, Bresnick GH, Kornguth SE. Paraneoplastic retinopathy associated with antiretinal bipolar cell antibodies in cutaneous malignant melanoma. Ophthalmology 1994;101:1236-43. 